\title{
When Is It Acceptable to Vaccinate Pregnant Women? Risk, Ethics, and Politics of Governance in Epidemic Crises
}

\author{
Ashley L. Graham ${ }^{1,2}$ \\ Published online: 18 October 2019 \\ (C) Springer Nature Switzerland AG 2019
}

\begin{abstract}
Purpose of Review In some countries, pregnant women experience disproportionately high morbidity and mortality during infectious disease outbreaks due to a variety of gender-based factors and pregnancy-related immunological changes. Despite this, the interests of pregnant women have largely been absent from policies that guide the design of clinical trials and the deployment of vaccines in epidemic contexts. This review examines historic precedent for both excluding and including pregnant women in vaccine trials and considers the rights of pregnant women in epidemic crises.

Recent Findings The latest research reveals that perceptions of risk and vulnerability of pregnant women in clinical research are beginning to change, resulting in modest policy and guideline amendments. A growing advocacy movement calling for "fair inclusion" has played an important role.

Summary Despite the global-scale and far-reaching implications of vaccine research policies, the current debate appears to reside primarily in disciplinary siloes across Western academic and policymaking spaces. Conceptual ambiguity of "risk," the pervasive view of pregnant women as "vulnerable," and competing ethical values that construct research protocols, globally, call for more explicit guidelines.
\end{abstract}

Keywords Pregnancy $\cdot$ Maternal immunization $\cdot$ Reproductive governance $\cdot$ Fair inclusion $\cdot$ Epidemic $\cdot$ Risk

\section{Introduction}

Major ecological shifts related to climate change and an unprecedented mobilization of people and goods have converged previously disparate human populations into a single "global disease ecology" [1]. This shift is marked by increasingly frequent and far-reaching epidemics of infectious disease, requiring more complex mechanisms of governance. The ongoing development, testing, and distribution of efficacious vaccines figure prominently in global health strategies to prepare for and respond to epidemics of emerging pathogens like Ebola, dengue, Marburg, hepatitis E, and Zika. With these diseases, it is well-documented that pregnant

This article is part of the Topical Collection on Vaccines in Pregnant Women \& Infants

Ashley L. Graham

University of Connecticut, Storrs CT USA

2 Atlanta USA women experience high morbidity and mortality during outbreaks due to increased gender-based exposure as caretakers of infected persons, involvement in funerary practices, and pregnancy-related immune suppression [2, 3]. However, another contributing factor to the suffering and death of pregnant and lactating women in epidemic contexts is their exclusion from clinical trials and emergency use of experimental vaccines [4]. The exclusion of pregnant women means that they have few-if any-options to protect themselves or their offspring from lifethreatening pathogens circulating in their households and communities. This review examines historic precedent for both excluding and including pregnant women in vaccine trials, explores the changing conceptions of "risk" and "vulnerability" that guide the vaccination of pregnant women, and considers the rights of pregnant women in epidemic crises.

\section{Exclusionary History}

In 1977, the U.S. Food and Drug Administration (FDA) released a guideline calling for the exclusion of "women with 
child-bearing potential," from the early phases of most clinical studies [5]. This policy came on the heels of the diethylstilboestrol (DES) and thalidomide tragedies in which both drugs, prescribed during pregnancy, were later linked to thousands of serious adverse complications, including the development of carcinomas and birth defects $[6,7]$. These incidents contributed greatly to the protectionist climate in clinical research today, in which any fetal risk is deemed unacceptable if there are no direct benefits to participating subjects. However, critics argue that the tragedies actually resulted from the dearth of safety and efficacy data precisely because the drugs were not tested in pregnant women $[8 \bullet \bullet]$. Today, with 9 out of 10 pregnant women in the USA taking some kind of medication, the overwhelming majority of which have "undetermined risk" in pregnancy [9]; prescribers are often "flying blind," unsure of safety profiles or proper dosing [10].

While pregnant women are sometimes included in research, the studies typically involve obstetric conditions, for which pregnant women and their offspring are the only affected populations. According to the Ethics Working Group on ZIKV Research and Pregnancy, "historically, the needs of pregnant women have not been adequately represented in the development of biomedical interventions, including vaccines" and "[n]ew products are rarely designed with the specific needs of pregnant women in mind" [11]. The "zero-risk" precautionary stance that guides research in pregnancy holds even in outbreak contexts where pregnant women and their offspring face extremely high mortality rates. Data from previous Ebola outbreaks indicate that the case fatality rate (CFR) for infected pregnant women can be as high as $93 \%$, and the CFR for fetuses of infected mothers is nearly $100 \%$ [4]. In documented cases of maternal infection with Marburg virus diseaseclosely related to Ebola virus disease (EVD) though much less common - all pregnant women and their infants who became infected had a fatal outcome [12]. According to the WHO Ethics Working Group, experimental therapeutics used in these emergency contexts must still undergo rigorous safety and efficacy trials [13]. While there are exceptions, such as "compassionate use" and "monitored emergency use of unregistered and experimental interventions" (MEURI), these exceptional uses do not typically apply to pregnant women [4].

Research involving pregnant women is complex, due to changing physiology and immunity throughout the weeks, months, and trimesters of pregnancy. However, the absence of safety and efficacy data for pharmacological interventions in pregnancy places pregnant women at greater risk by ensuring a sizable gap in knowledge that leads to greater health inequity downstream [14]. In light of this, a growing number of advocates have called for the inclusion of pregnant women in research, including ethicists, pharmacologists, social scientists, researchers, and regulators.

The shift toward fair inclusion of pregnant women began in the early 1990s, with the endorsement of the "presumed eligibility" of pregnant women in clinical research by the U.S. Department of Health and Human Services [8••, 15]. In 2002, the Council for International Organizations of Medical Sciences (CIOMS) - formed by the WHO to guide ethical, international biomedical research - adopted this "eligibility claim" and revised its guidelines accordingly [16]. However, until recently, policy change has been modest. What has changed in the last decade is the proliferation of advocacy efforts and calls for research to address the needs of pregnant women. This includes the formation of several specialized working groups, including the Second Wave Initiative [17], PHASES (Pregnancy and HIV/AIDS: Seeking Equitable Study) [18], PREVENT (Pregnancy Research Ethics for Vaccines, Epidemics, and New Technologies) [19•], and the Ethics Working Group on ZIKV Research and Pregnancy $[19 \cdot, 20]$. These initiatives aim to examine ethical and scientifically sound pathways to build an evidence base for pregnant women. While the resulting reports and recommendations have encouraged greater dialogue about the issue, most research protocols and entrenched perceptions of research in pregnancy remain largely unchanged.

The U.S. National Institutes of Health (NIH) have supported vaccine studies allowing the participation of pregnant women since the 1980s [21]. However, most have been observational and involved interventions for obstetric conditions or conditions affecting neonates and infants, not conditions common in the general population. With changing recommendations for vaccines in pregnancy-including widespread support and use of tetanus, influenza, and pertussis vaccines in pregnant women - several recent vaccine trials have proactively included pregnant women. These trials for group B streptococcus (GBS), respiratory syncytial virus (RSV), and Streptococcus pneumoniae have shown good safety and immunogenicity [21-23]. While these trials signal a willingness to include pregnant women in research, it is important to note that these vaccines treat conditions that predominantly impact pregnant women and infants. Generally, trials for vaccines that protect against infectious diseases with outbreak potential have not included pregnant women.

\section{Push for Inclusion in Outbreaks}

The debate over inclusion of pregnant women in vaccine research is becoming more urgent and contentious as the pace of vaccine development accelerates and increasingly frequent outbreaks are shown to be highly virulent for pregnant women and their unborn babies, with some-like Zika - causing debilitating sequelae. While the debate extends well beyond epidemic context, examination of the issue in outbreak settings is particularly instructive. According to Schwartz, "when creating policy about these issues, it is important to critically evaluate vaccine safety in pregnancy in the context of the 
substantial risk of infection for the pregnant woman and her fetus in the absence of immunization" (emphasis added) [24•].

When Ebola cases began to appear in North Kivu in the Democratic Republic of Congo (DRC) in the summer of 2018, the Ministry of Health moved to begin ring vaccination with the recombinant vesicular stomatitis virus-Zaire Ebola virus (rVSV-ZEBOV) Merck vaccine. The vaccine was tested in previous outbreaks and was shown to offer substantial protection against EBV in observational studies and randomized control trials [25]. When vaccination began on August 8, the Ministry of Health, with support from the WHO and other partners, decided that pregnant and lactating women would be excluded from reciving the vaccine [24•]. Shortly after this controversial decision, members of the PREVENT initiative wrote an opinion piece imploring the DRC Ministry of Health and the WHO to recognize that pregnant and lactating women should have equitable access to the lifesaving benefits of the vaccine. They wrote, "[f]rom a public health perspective and an ethical perspective, the decision to exclude pregnant and lactating women is utterly indefensible" [26]. These experts acknowledged that the vaccine may be inappropriate for use in pregnant women outside of an outbreak context, but that the "catastrophic maternal and fetal mortality rates [in outbreak] change the calculus."

Reluctance to offer pregnant women the Ebola vaccine is rooted in the fact that it is a live-virus vaccine, and live-virus formulations are typically not recommended for pregnant women due to "theoretical risk" to the fetus. However, there is precedent for recommending the use of live vaccines and other treatments thought to pose a "theoretical risk" during epidemics. During the AIDS pandemic in the 1980s, for example, the recognition of perinatal transmission of HIV provided researchers grounds to enroll pregnant women in early phases of anti-retroviral drug trials [24•]. According to Schwartz, "the life-threatening nature of AIDS was believed to justify an unknown risk to the fetus in order to potentially extend the life of the mother" [24•]. More recently, the WHO reversed its recommendation on the yellow fever vaccine, encouraging its use in pregnant women during outbreaks, expanding on the manufacturer's labeling, which merely states "YF-VAX should be given to pregnant women only if clearly needed" (emphasis added) [27]. According to the WHO, "during outbreaks, the benefits of vaccination are likely to far outweigh the risk of potential transmission of [yellow fever] vaccine virus to the fetus or infant" [28].

In February 2019, 7 months after the North Kivu outbreak began, officials in the DRC announced the decision to offer women who are pregnant and lactating access to the Merck vaccine, marking a controversial policy reversal [29]. According to Carleigh Krubiner, a policy expert and member of the PREVENT initiative, "The DRC's decision to extend Ebola vaccine coverage to pregnant women is a huge step forward, not only for pregnant women in areas affected by outbreaks but for all pregnant women who may face the threat of Ebola in the future" [29].

The Strategic Advisory Group of Experts (SAGE) on Immunization, which advises the WHO, recently stressed the importance of collecting data to inform future policy on safety and effectiveness of vaccines in pregnant and lactating women [29]. The safety of the rVSV-ZEBOV vaccine in pregnant women remains to be seen, as published data is limited given the recency of their inclusion. Additionally, limited knowledge of background rates of adverse events in pregnancy could make safety assessments difficult [30].

While efforts to contain the latest outbreak of Ebola in the DRC are ongoing, efforts to develop a Zika vaccine are also underway. More than $45 \mathrm{Zika}$ vaccine candidates are in development, some in phase 2 clinical trials [31], and advisory groups and regulatory bodies are proactively considering the importance of testing in pregnancy. The latest version of the WHO Target Product Profile for Zika Vaccines in Outbreak (TPP) states that "[t]heoretical risk may not preclude the exceptional use during pregnancy or in lactating women during an outbreak" and "the immunization of women of reproductive age, which may include pregnant women, is considered to be of highest priority" [32]. To date, however, no pregnant women are enrolled in ongoing Zika vaccine trials.

\section{Changing Perspectives on "Risk" and "Vulnerability"}

Some scholars criticize the discourse on research in pregnancy for dichotomizing risk into "dangerous" and "safe" [33], which is a reductionist perspective not applied to research in the general population. Unfortunately, given the conceptual ambiguity of "risk," coupled with entrenched perceptions of "vulnerability" in pregnancy and a lack of clear and instructive guidelines, the risk-benefit analyses conducted in vaccine research impede progress toward the fair inclusion of pregnant women.

Clinical trials in emergency contexts are inherently difficult to plan and execute, often raising more complex ethical issues around risk [34]. A major challenge is the conceptual haziness of "acceptable risk" to a woman or fetus. According to the HHS policy guiding research with human subjects-also known as the "Common Rule" - if trial participation will not directly benefit the woman or fetus, risk must not be "greater than minimal harm" [35••]. Unfortunately, "minimal risk" is simply not well-defined for research in pregnancy [36]. The Common Rule also states that harms anticipated in research cannot be greater "than those ordinarily encountered in daily life" [37]. This particular phrasing raises an important question. How does outbreak context change the risk-benefit analysis of vaccines in pregnancy? In other words, since the risk and harms of "daily life" during an outbreak are likely 
much higher than in "normal" times, how does the risk calculus change to account for this? The WHO Ethics Review Committee (WHO-ERC), which reviewed two dozen research protocols for Ebola vaccine research during the West African outbreak (2014-2016), defined "benefit" as likely to "improve the chances of survival or reduce the probability of infection" and "risk" as resulting in "serious temporary or irreversible adverse reactions, including death" [34]. Given the high maternal and fetal mortality rate associated with Ebola infection, the potential benefit of intervention appears to outweigh the unknown risk.

The uncertainty around risk makes it exceedingly difficult to ensure fair inclusion of pregnant women in vaccine research during epidemics. The decision necessarily depends on the nature of the infectious disease itself. For example, is the risk to fetus viewed differently for an experimental Zika vaccine than it is for Ebola? If so, how do researchers and ethics review boards differentially weigh the benefits? Schopper and colleagues [38] offer a list of explicit questions to consider when evaluating the risks and benefits to determine if exclusion is justified (see Table 1).

A second barrier to fair inclusion of pregnant women is the categorization of pregnant women as "vulnerable" in research. The classification as "vulnerable to coercion or undue influence" situates pregnant women alongside children, prisoners, handicapped individuals, and economically or educationally disadvantaged persons [39]. According to the American College of Obstetricians and Gynecologists (ACOG), "pregnant women have the same capacity for autonomous decision-making as their nonpregnant counterparts" [14]. Advocates condemn this classification, calling it deeply problematic, and push for the use of "scientifically complex" to more accurately describe a pregnant research population [9, $40,41]$. As Heyrana and colleagues argue, "[p]regnant women are fully able to weigh the ethical implications of health decisions they make for themselves and their fetuses, especially when given adequate counseling about their conditions and treatment options" [35••].

Recently, policy documents have finally begun to reflect changing views on vulnerability thanks in large part to the initiatives advocating for fair inclusion of pregnant women. The 2016 revision of the CIOMS guidelines, for example, now asserts that "pregnant women must not be considered vulnerable simply because they are pregnant" [16]. The
CIOMS revision also laid the groundwork for the elimination of the designation of pregnant women as "vulnerable" in the Common Rule last year [37]. This amendment signals that pregnancy itself is no longer considered a justification for exclusion. However, the removal of the term "vulnerable" does not necessarily equate to more equitable inclusion, nor does it guarantee that pregnant women can exercise autonomy in the research process. According to a WHO staffer in Goma, DRC, henceforth referred to by the pseudonym Alana, pregnant women were still unable to access the experimental Ebola vaccine months after the Ministry of Health's official policy reversal (personal communication, April 2019). Today, pregnant women continue to be excluded from ongoing Ebola studies, including the phase 2 trial of a new candidate Ebola vaccine (Ad26.ZEBOV/MVA-BN®-Filo), initiated by the MRC/UVRI Uganda Research Unit on AIDS in August 2019 [42]. In this trial, both "pregnancy" and "plans to become pregnant" are exclusion criteria.

\section{Risk Epistemologies and Ethical Frameworks}

When is it acceptable to vaccinate pregnant women in outbreaks and other humanitarian crises? Framing in terms of "acceptability" can illuminate complex governance mechanisms and underlying logics at work in transnational vaccine research. What does "acceptable" mean in global health? What epistemologies and ethical frameworks guide this determination? The Ethics Working Group on ZIKV Research and Pregnancy offers the following insight: "[b]y acceptable for use in pregnancy we mean that relevant advisory bodies, public health practitioners, and policymakers could support the use of such a vaccine by pregnant women in an outbreak setting based on the expected benefits associated with the vaccine and its safety profile" [11]. In the absence of cohesive international policy, acceptability is interpreted and governed differentially across political and economic spheres, leading to what Petryna calls "ethical variability" [43]. In the debate over the inclusion of pregnant women in vaccine research, myriad risk epistemologies are at work, informed by profession, precedent, geography, and social context.

Generally speaking, "risk" refers to uncertainty about and severity of the consequences (or outcomes) of an activity with respect to something that humans value [44]. Different
Table 1 Key questions for evaluating risks and benefits of vaccines in pregnancy

\begin{tabular}{ll}
\hline $\begin{array}{l}\text { What are the clinical outcomes for } \\
\text { pregnancies in this context? }\end{array}$ & What is the average time from infection/diagnosis to mortality? \\
\hline Is it altered during pregnancy? & $\begin{array}{l}\text { Do the criteria for exclusion include a balance of risks and benefits } \\
\text { for both the fetus and the mother? }\end{array}$ \\
What phase is the study? & Are there any existing safety data for the intervention? \\
What other interventions are available? & Whose interests should be considered in our determination?
\end{tabular}


disciplines employ various techniques and abstractions to interpret and explain risk. In science and medicine, risk is an objective reality that can be measured and controlled, and the knowledge applied to risk is based upon calculations, principles, and postulates [45]. It is within this frame that the notion "acceptable" risk was first articulated [46]. In anthropology, cultural frameworks and social constructs explain risk, highlighting the fallacy of science's propensity to think of risk as "objective," insisting instead that risk is an inherently moral classification [47]. In this view, there is no single arbiter of what is risky. It is a political and moral evaluation filtered through shared expectations and norms [48]. Therefore, in risk analysis, it is important to focus not only on the perceived dangers presented in a given situation (e.g., the teratogenic effects of a novel vaccine) but also on the way they are politicized and moralized. When presented with the notion of "theoretical risk" to the fetus as ethical, scientific, and legal grounds for exclusion of pregnant women in vaccine studies, decision-makers draw on several sets of cultural norms and expectations. According to Timmermans and Buchbinder, "[m]edical uncertainty has a deep moral and existential dimension: it provokes fundamental questions about whether lives are worth living" [49]. The valuation-which life is worth living - is particularly salient in this debate, as the lives of the mother and fetus are often framed as being at odds, while they are, in reality, deeply intertwined and their interests typically aligned.

Risk calculation is inherently ethical, since weighing risk against benefit is guided by "principles and cultural rules" that determine what is considered acceptable [50]. In biomedical research, the principles are laid out explicitly in guidelines (e.g., The Belmont Report, CIOMS) and regulated by ethics review boards and national policy. The cultural rules, however, are less visible and often unexamined. According to the WHO, "[a]n ethically acceptable decision depends on articulating the full range of appropriate ethical considerations, ensuring that multiple perspectives are factored into the analysis" [51]. But which perspectives are included? Does the "full range" of considerations encompass how pregnant women weigh risks? According to Heyrana and colleagues:

how a woman or family defines reasonable risk may differ depending on their view of the anticipated opportunities and expectations for the pregnancy and future baby, as well as the urgency of the clinical question. In some circumstances, pregnant women may tolerate uncertainty when the risk is high. [35••]

The aforementioned WHO staffer, Alana, echoed this view. She shared that, even when they were excluded from Ebola vaccine trials, pregnant women in North Kivu began to actively seek the vaccine. As she explained, the women believed the danger of contracting Ebola in their community was much greater than the unknown risks of the vaccine to themselves and their babies. The risk calculations of pregnant women, in this case, included factors not captured in biomedical research spaces. They thought not only about the risk to their unborn child but also about the wellbeing of their other children, who relied on them for care. This example clearly highlights what Kleinman calls the "dangerous break" between bioethics and local moral worlds [52].

\section{Reproductive Governance and Social Justice}

Transnational vaccine research in epidemic context is a multifaceted and complex "global assemblage" [53], which brings together ministries of health, national health organizations (e.g., FDA, NIH), pharmaceutical companies, bilateral organizations, international institutions like the WHO, and nonprofit organizations like Médecins Sans Frontières (MSF). Within this amalgam is a multitude of competing epistemologies and ontological assumptions. Vaccine developers are at once concerned with evidence-based medicine and legal liability, while the WHO engages with both global health security [54] and health equity. These contradictions make the design of research protocols and the global health policies that guide them fraught with contradiction. Using "governance" as an interpretive framework reveals how vaccine trials that exclude pregnant women are shaped by particular beliefs about reproduction and individual rights.

According to ACOG, "[i]t is important that researchers and funding organizations recognize the ways in which fertility, in the context of research trials, has been managed historically in a manner that is not evidence-based and that is overly burdensome" [14]. In anthropology, the concept "reproductive governance" is instructive [55-57]. Morgan and Roberts define reproductive governance as follows:

the mechanisms through which different historical configurations of actors - such as state, religious, and international financial institutions, NGOs, and social movements - use legislative controls, economic inducements, moral injunctions, direct coercion, and ethical incitements to produce, monitor, and control reproductive behaviours and population practices. [55]

Analysis of reproduction as a culturally situated phenomenon allows a window into the complex configurations of biomedicine, technology, and politics that regulate women's bodies [58]. Viewed this way, the regulation of women's participation in research reifies the long-standing tension between the "right-to-life" of the unborn and the autonomy of women. Despite the fact that maternal and fetal risks are deeply 
intertwined, risk is typically framed as dichotomous [8••, 40], which only serves to deepen the perspective that fetal and maternal interests are at odds in research.

At stake in this debate, though less robustly addressed in the literature, are the rights of pregnant women. The violation of rights is twofold. First, the unjustifiable exclusion of pregnant women from research participation denies them the autonomy to choose, which is typically exercised through an informed consent process. Pregnant women are also denied the potential benefits of trial participation, and - in the case of deadly epidemics - may be subjected to suffering and death as a result. Alirol and colleagues call these "individual injustices" [34]. However, the exclusion of pregnant women as a group results in a second order of injustice, a "social injustice." This second-order injustice guarantees the absence of safe and effective interventions for pregnant women and infants during future epidemics, leading to downstream inequities and diminishing the potential value of clinical trials [34].

\section{Conclusions}

Despite the global-scale and far-reaching implications of vaccine research policies, the current debate around the inclusion of pregnant women appears to reside primarily in disciplinary siloes across academic and policymaking spaces, where Western biomedical perspectives are privileged, and the voices of pregnant women are conspicuously absent. Entrenched biomedical research norms and perspectives of risk and vulnerability will prove difficult to change unless we confront embedded ethical claims, politics of governance, risk calculus, and accepted forms of knowledge that construct vaccine research policies in epidemic contexts and beyond.

This review outlines changing perceptions of risk and vulnerability, recent relevant policy and guideline amendments, and the contributions of scholars advocating for fair inclusion. It also calls attention to the tensions that exist between underlying ethical values and perceptions of risk, and the ways in which the governance of vaccine trials limits the rights and autonomy of pregnant women. Conceptual ambiguity of "risk," the pervasive nature of the view of pregnant women as "vulnerable," and competing ethical values that construct research protocols, globally, call for more explicit guidelines.

Drawing solely on the principles of clinical ethics and biomedical research ethics is insufficient to address the complexity and scope of global health research in epidemic contexts. At present, the nascent field of "global health ethics" lacks a cohesive framework for navigating ethical dilemmas such as those illustrated here [59]. Further research is needed to examine the risk epistemologies, ethical frameworks, and mechanisms of governance that determine the acceptability of including pregnant women in research. Such an inquiry would enable us to grapple with important questions such as (1) What are the unintended consequences of applying broad biomedical notions of risk in pregnancy across time and space? [58] and (2) How might local experiences and norms of maternal immunization and reproduction, as well as definitions of health, personhood, and/or death, shape research design? [60].

At present, pregnant women do not appear to have a say in the debate over their inclusion in research. The production of scientific knowledge about risk in pregnancy and dominant discourses around reproduction and ethical conduct work together to reinforce unexamined norms in vaccine research that regulate women's bodies [58]. Moving forward, women must be represented in decision-making spaces that influence both national and international policies that guide maternal immunization [61]. In doing so, we may more meaningfully engage with issues beyond "risk" and "vulnerability" to better address questions of agency, equity, and social justice.

\section{Compliance with Ethical Standards}

Conflict of Interest The author declares that she has no conflict of interest.

Human and Animal Rights and Informed Consent This article does not contain any studies with human or animal subjects performed by any of the authors.

\section{References}

Papers of particular interest, published recently, have been highlighted as:

- Of importance

•- Of major importance

1. Barrett R, Kuzawa CW, McDade T, Armelagos GJ. Emerging and re-emerging infectious diseases: the third epidemiologic transition. Annu Rev Anthropol. 1998;27:247.

2. Menendez C, Lucas A, Munguambe K. Ebola crisis: the unequal impact on women and child's health. Lancet. 2015;e130. https:/ doi.org/10.1016/S2214-109X(15)70009-4.

3. Bebell LM, Oduyebo T, Riley LE. Ebola virus disease and pregnancy - a review of the current knowledge of Ebola virus pathogenesis, maternal and neonatal outcomes. Birth Defects Res. 2017;109(5):353-62. https://doi.org/10.1002/bdra.23558.

4. Caluwaerts S. Nubia's mother: being pregnant in the time of experimental vaccines and therapeutics for Ebola. Reprod Health. 2017;14(3):157. https://doi.org/10.1186/s12978-017-0429-8.

5. Merkatz R. Inclusion of women in clinical trials: a historical overview of scientific, ethical, and legal issues. JOGNN. 1998;27(1): $78-84$.

6. Allesee L, Gallagher CM. Pregnancy and protection: the ethics of limiting a pregnant woman's participation in clinical trials. J Clin Res Bioeth. 2011;2(108).

7. Swan SH. Intrauterine exposure to diethylstilbestrol: long-term effects in humans. APMIS. 2000;108:793-804.

8.• van der Graaf R, van der Zande ISE, van Delden JJM. How the CIOMS guidelines contribute to fair inclusion of pregnant women in research. Bioethics. 2019;33:377-83. https://doi.org/10.1111/ 
bioe. 12520 van der Graaf and colleagues trace the revision of CIOMS guidelines since 2002, revealing recent changes that foster greater inclusion of pregnant women in research.

9. Krubiner CB, Faden RR. Pregnant women should not be categorised as a 'vulnerable population' in biomedical research studies: ending a vicious cycle of 'vulnerability'. J Med Ethics. 2017;43(10):664-5. https://doi.org/10.1136/medethics-2016103955.

10. Rubin R. Addressing barriers to inclusion of pregnant women in clinical trials. JAMA. 2018;320(8):742-4.

11. The Ethics Working Group on ZIKV Research and Pregnancy. Ethics, pregnancy, and ZIKV vaccine research \& development. Vaccine. 2017;35:6819-22. https://doi.org/10.1016/j.vaccine. 2017.09.065.

12. Schwartz DA. Maternal filovirus infection and death from Marburg and Ravn viruses: highly lethal to pregnant women and their fetuses similar to Ebola virus. In: Okware SI, editor. Re-emerging filovirus diseases. London: IntechOpen; 2019. Available from: https://www. intechopen.com/online-first/maternal-filovirus-infection-anddeath-from-marburg-and-ravn-viruses-highly-lethal-to-pregnantwomen. Accessed 1 Sept 2019.

13. WHO Ethics Working Group. Ethical issues related to study design for trials on therapeutics for Ebola virus disease: WHO Ethics Working Group Meeting 20-21 October, Summary of Discussion. 2014. http://apps.who.int/iris/handle/10665/137509. Accessed 1 Aug 2019.

14. American College of Obstetricians and Gynecologists. ACOG Committee Opinion No. 646: ethical considerations for including women as research participants. Obstet Gynecol. 2015;126(5): e100-7 Reaffirmed 2018.

15. U.S. Department of Health and Human Services, National Institutes of Health. NIH guidelines on the inclusion of women and minorities as subjects in clinical research. Fed Regist. 1994:59:14508-13.

16. Council for International Organizations of Medical Sciences (CIOMS). International ethical guidelines for health-related research involving humans. Geneva, Switzerland; 2002. Available from: http://cioms.ch/ethical-guidelines-2016/WEB-CIOMSEthicalGuidelines.pdf. Accessed 5 Jun 2019.

17. Lyerly AD, Little MO, Faden R. The second wave: toward responsible inclusion of pregnant women in research. IJFAB: Int J Fem Approaches Bioeth. 2008;1(2):5-22. The Second Wave Initiative. Retrieved from http://secondwaveinitiative.org/. Accessed 28 Aug 2019.

18. PHASES. Pregnancy and HIV/AIDS: Seeking Equitable Study. http://www.hivpregnancyethics.org/. Accessed 25 June 2019.

19. The PREVENT Working Group. Pregnant women \& vaccines against emerging epidemic threats: ethics guidance for preparedness, research, and response. Baltimore, MD; 2018. Developed by a multidisciplinary, international team of experts, the PREVENT working group offers guidance for vaccine research and development to ensure the needs of pregnant women and their offspring are ethically and fairly addressed.

20. Little MO, Lyerly AD, Faden RR. Pregnant women and medical research: a moral imperative. Bioeth Forum. 2009;2:60-5.

21. Jones CE, Munoz FM, Spiegel HML, Heininger U, Zuber PLF, Edwards KM, et al. Guideline for collection, analysis and presentation of safety data in clinical trials of vaccines in pregnant women. Vaccine. 2016;34(49):5998-6006.

22. Marshall H, McMillan M, Andrews RM, Macartney K, Edwards $\mathrm{K}$. Vaccines in pregnancy: the dual benefit for pregnant women and infants. Hum Vaccin Immunother. 2016;12(4):848-56. https://doi. org/10.1080/21645515.2015.1127485.

23. Cohen J. Zika rewrites maternal immunization ethics. Science. 2017;357(6348):241. https://doi.org/10.1126/science.357.6348. 241.
24. Schwartz DA. Clinical trials and administration of Zika virus vaccine in pregnant women: lessons (that should have been) learned from excluding immunization with the Ebola vaccine during pregnancy and lactation. Vaccines. 2018;6(4). https://doi.org/10.3390/ vaccines6040081. Schwartz analyzes the exclusion of pregnant women in West Africa and the DRC from receiving the lifesaving Ebola vaccine and discusses important implications for Zika vaccine trials.

25. Henao-Restrepo AM, Longini IM, Egger M, Dean NE, Edmunds WJ, Camacho A, et al. Efficacy and effectiveness of an rVSVvectored vaccine in preventing Ebola virus disease: final results from the Guinea ring vaccination, open-label, cluster-randomized trial (Ebola Ca Suffit!). Lancet. 2017;389:505-18. https://doi.org/ 10.1016/S0140-6736(16)32621-6.

26. Faden, R, Karron R, Krubiner C. An 'indefensible' decision: not vaccinating pregnant and lactating women in an Ebola outbreak. STAT News August 27, 2018. https://www.statnews.com/2018/08/ 27/ebola-vaccine-pregnant-lactating-women/. Accessed 28 July 2019

27. Yellow Fever Vaccine (YF-VAX) product information. Sanofi Pasteur, June 2016. Accessed July 15, 2019.

28. WHO yellow fever fact sheet. https://www.who.int/ith/vaccines/yf/ en/. Accessed 15 July 2019.

29. Branswell H. Ebola vaccine will be provided to women who are pregnant, marking reversal in policy. STAT News. February 20, 2019. https://www.statnews.com/2019/02/20/ebola-pregnancyreversal/. Accessed 25 July 2019.

30. Jones CE, Calvert A, Le Doare K. Vaccination in pregnancy recent developments. Pediatr Infect Dis J. 2018;37(2):192.

31. Wilder-Smith A, Vannice K, Durbin A, Homach J, Thomas SJ, Thevarjan I, et al. Zika vaccines and therapeutics: landscape analysis and challenges ahead. BMC Med. 2018;16(84). https://doi.org/ 10.1186/s12916-018-1067-x.

32. WHO, UNICEF. WHO/UNICEF Zika Virus (ZIKV) Vaccine Target Product Profile (TPP): vaccine to protect against congenital Zika syndrome for use during an emergency. Released July 2016. Updated February 2017. http://www.who.int/immunization/ research/development/zika/en/.

33. Ballantyne A, Pullon S, Macdonald L, Barthow C, Wickens K, Crane J. The experiences of pregnant women in an interventional clinical trial: Research in Pregnancy Ethics (RIPE) study. Bioethics. 2017;31:476-83. https://doi.org/10.1111/bioe.12361.

34. Alirol E, Kuesel AC, Guraiib MM, de la Fuente-Núñez V, Saxena A, Gomes MF. Ethics review of studies during public health emergencies - the experience of the WHO ethics review committee during the Ebola virus disease epidemic. BMC Med Ethics. 2017;18:43. https://doi.org/10.1186/s12910-017-0201-1.

35.• Heyrana K, Byers HM, Stratton P. Increasing the Participation of Pregnant Women in Clinical Trials. JAMA. 2018;320(20):2071. https://doi.org/10.1001/jama.2018.17716 Heyrana et al. outline three specific barriers that impede fair inclusion of pregnant women in vaccine trials: their designation as "vulnerable," ambiguity around "acceptable risk," and legal liability. They call for the appropriate engagement of pregnant women and their fetuses in clinical research to not only improve their health but also develop the evidence base.

36. Omer SB, Beigi RH. Pregnancy in the time of Zika: addressing barriers for developing vaccines and other measures for pregnant women. JAMA. 2016;315(12). https://doi.org/10.1001/jama.2016. 2237.

37. Office for Human Research Protections. Revised common rule. Rockville: Office for Human Research Protections; 2018.

38. Schopper D, Ravinetto R, Schwartz L, Kamaara E, Sheel S, Segelid M, et al. Research ethics governance in times of Ebola. Public Health Ethics. 2017;10(1):49-61 Accessed July 31, 2019. 
39. Rubin R. Addressing barriers to inclusion of pregnant women in clinical trials. JAMA. 2018;320(8). Accessed July 31, 2019.

40. Blehar MC, Spong C, Grady C, Goldkind SF, Sahin L, Clayton JA. Enrolling pregnant women: issues in clinical research. Womens Health Issues. 2013;23:e39-45.

41. White A. Accelerating the paradigm shift toward inclusion of pregnant women in drug research: ethical and regulatory considerations. Semin Perinatol. 2015:537-40. https://doi.org/10.1053/j.semperi. 2015.08.008

42. David A. Schwartz, personal communication, August 2019.

43. Petryna A. Ethical variability: drug development and globalizing clinical trials. Am Ethnol. 2005;32(2):183-97.

44. Aven T, Renn O. On risk defined as an event where the outcome is uncertain. J Risk Res. 2009;12(1):1-11.

45. Adams J. Risk. London: UCL Press; 1995.

46. Baker B. Risk assessment kills bills. Bioscience. 1995;45(1):15.

47. Douglas M. Risk and blame. London: Routledge; 1992.

48. Althaus C. A Disciplinary perspective on the epistemological status of risk. Risk Anal. 2005;25(3):567-88. https://doi.org/10.1111/j. 1539-6924.2005.00625.x.

49. Timmermans S, Buchbinder M. Saving babies? The consequences of newborn genetic screening. Chicago: University of Chicago Press; 2013. p. 13.

50. Stewart K. Anthropological perspectives in bioethics. Int Encycl Publ Health. 2017;1:113 Accessed June 14, 2019.

51. World Health Organization (WHO). Ethics guidance: for the implementation of the end TB strategy 2017. Geneva, Switzerland.

52. Kleinman A. Moral experience and ethical reflection: can ethnography reconcile them: a quandary for "the new bioethics" Daedalus. 1999;128(4):69-97.
53. Collier SJ, Ong A. Global assemblages, anthropological problems. In: Ong A, Collier SJ, editors. Global assemblages: technology, politics, and ethics as anthropological problems. Malden: Blackwell; 2005. p. 3-21.

54. Lakoff A. Unprepared: global health in a time of emergency. Oakland: University of California Press; 2017.

55. Morgan LM, Roberts EFS. Reproductive governance in Latin America. Anthropol Med. 2012;19(2):241-54. https://doi.org/10. 1080/13648470.2012.675046.

56. Ginsburg F, Rapp R. The politics of reproduction. Annu Rev Anthropol. 1991;20:311-43.

57. Ginsburg F, Rapp R. Conceiving the new world order: the global politics of reproduction: University of California Press; 1995.

58. Hallowell E. "Between the wall and the sword": reproductive governance and the technology of emergency in Guatemalan maternity care. Fem Form. 2014;26(3):100-21.

59. Addiss DG, Amon JJ. Apology and unintended harm in global health. Health Hum Rights. 2019;21(1):19-32.

60. Feierman S, Kleinman A, Stewart K, Farmer P, Das V. Anthropology, knowledge-flows, and global health. Glob Publ Health. 2010;5:122-8.

61. Chamberlain AT, Lavery JV, White A, Omer SB. Ethics of maternal vaccination: involvement of women is critical in establishing guidelines. Sci Mag. 2017;358(6362):453 http://science.sciencemag.org/ Accessed 25 June 252019.

Publisher's Note Springer Nature remains neutral with regard to jurisdictional claims in published maps and institutional affiliations. 